\title{
EFFECT OF NITROGEN SOURCES AND ASCORBIC ACID ON GROWTH AND ESSENTIAL OIL PRODUCTION OF GERANIUM (PELARGONIUM GRAVEOLENS, L.) PLANTS
}

\author{
M.A.H. Abdou ; E.E.T. Ahmed ${ }^{*}$ and T.I.E. Ibrahim ${ }^{* *}$ \\ * Hort. Dept., Fac. Agric., Minia Univ., Egypt. \\ ** Ministry of Agriculture, Egypt.
}

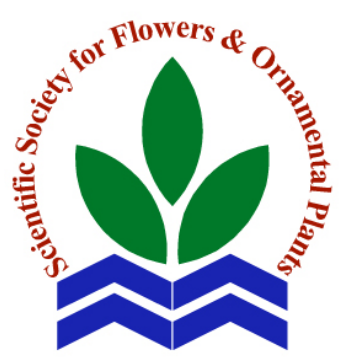

Scientific J. Flowers \& Ornamental Plants, 2(2):157-165 (2015).

Received:

23/6/2015

Revised by:

Prof. Dr. E.S. Nofal, Kafr El-Sheikh Univ.

Prof. Dr. M.S. Hanafy, Cairo Univ.

ABSTRACT: The experiment was conducted during two successive seasons (2012/2013 and 2013/2014) in the nursery of ornamental plants and the Laboratory of Floriculture, Fac. of Agric., Minia Univ. The aim of this work is to study the effect of bio-mineral nitrogen and ascorbic acid (50, 100 and $150 \mathrm{ppm})$ on growth and essential oil production of geranium plants. Results showed that non-significant differences between mineral N (100\%) and mineral N (75\%) + Minia Azotein (Azotobacter strain) on growth and essential oil production of plants. The effect of high concentration $(150 \mathrm{ppm})$ of ascorbic acid was superior to that other two concentrations (medium and low) on increasing plant growth and essential oil productivity.

The plants fertilized with mineral N (75\%) plus Minia Azotein (Azotobacter strain) and spraying with $150 \mathrm{ppm}$ ascorbic were equal (growth and essential oil production) with plants received $100 \% \mathrm{~N}$ plus 150 ppm ascorbic acid.

Key words: Pelargonium graveolens, bio-mineral nitrogen, ascorbic acid, vegetative growth, essential oil.

\section{INTRODUCTION}

Pelargonium graveolens, L. belongs to family Geraniaceae. It is not to be confused with the household variety of geranium, which is a completely different species. There are over 700 varieties of cultivated geranium, however, most are grown for ornamental purposes. The plants of Pelargonium are native to South Africa (Shawi et al., 2006). In warmer climates like that of Egypt, the plants are growing as a perennial, lasting on the average from four to six years (Kaul et al., 1996).

Geranium oil can range from very sweet and rosy to musty, minty and green (Rejeshwara Rao, 2002). Its action include analgestic, antidepressant, antiseptic, astringent, cicatrisant, cytophylactic, deodorant, styptic, tonic, vulnerary (Qinghua, 1993).
Chemical nutrients especially nitrogen is very important for plants, because $\mathrm{N}$ partake in structure of several components of the whole plant. Application of mineral fertilization have been reported to enhance plant growth and to increase the active ingredient content in several medicinal plants such as geranium (Ram et al., 2003), Ocimum basilicum (Golcz et al., 2006), lemon Balm (Aziz and El-Ashry, 2009) and peppermint (Zheljazkov and Cerven, 2009).

Biofertilizers are considered to be low cost, eco-friendly and renewable sources of plant nutrients supplementing chemical fertilizers in sustainable agricultural system. This refer to microorganism, which increase crop growth through different mechanisms, i.e. biological nitrogen fixation, growth promoting or hormonal substances, increasing availability of soil nutrients (Galal and Aly, 2004). Application Minia Azotein 
as biofertilizer increased plant growth and essential oil production in several plants such as anise (Hemdan, 2008), black cumin (Helmy, 2008), borage plant (Abdou et al., 2009) and sweet basil (Abdou et al., 2014).

Ascorbic acid (vit. C) had many stimulating effects on growth and some physiological activities of different plant (Dewick, 2000; Ismail, 2008 and Abdou et al., 2009).

This object of this study is to investigate the response of geranium plants to different levels of mineral nitrogen with biofertilizer (Minia Azotein) and ascorbic acid, as well as, their interaction on vegetative growth and essential oil yield in order to figure out the possibility of decreasing the amount of mineral nitrogen by the substitution with biofertilizers.

\section{MATERIALS AND METHODS}

A field experiment was conducted during the two successive growing seasons of 2012/2013 and 2013/2014 at the nursery of ornamental plants and the Laboratory of Floriculture, Fac. of Agric., Minia Univ. to investigate the effect of different concentrations of mineral nitrogen with Minia Azotein (Azotobacter strain) and ascorbic acid, as well as, their interaction treatments on vegetative growth traits and essential oil productivity of Pelargonium graveolens, $\mathrm{L}$.

The uniform terminal cuttings of geranium were taken yearly from the mother plants in uniform size and length $(18 \mathrm{~cm})$ long. The cuttings were immediately planted in polyethylene bags in a mixture of clay: sand $(1: 1 \mathrm{v} / \mathrm{v})$ on November $24^{\text {th }}, 2012$ and 2013, respectively. The seedlings were transplanted in the Experimental filed on February $20^{\text {th }}$ for each season.

The experiment was arranged in a splitplot design with three replicates. The main plots included three levels of mineral $\mathrm{N}$ plus Minia Azotein (A), while, four treatments of ascorbic acid (B) occupied the sub plots. Therefore, the interaction treatments $(\mathrm{A} \times \mathrm{B})$ were 16 treatments. The experimental unit (plot) was $3 \times 3 \mathrm{~m}$ and containing 4 rows, 60 $\mathrm{cm}$ apart and seedlings were cultivated in hills, 32 plants. The physical and chemical analysis of the soil are shown in Table (a):

\section{Treatments:}

1- Main plots were mineral N 25\% + Minia Azotein (M.A.), mineral N 50\%+ Minia Azotein, mineral N 75\% + Minia Azotein and mineral N 100\% (recommended dose). Mineral (full dose) was added at the rate of $400 \mathrm{~kg} / \mathrm{fed} / \mathrm{year}$ of ammonium nitrate $(33.5 \% \mathrm{~N})$. So the mineral $\mathrm{N}$ at 25,50 and $75 \%$ were 100, 200 and $300 \mathrm{~kg} /$ fed/year.

All plants received mineral $\mathrm{PK}$ as calcium superphosphate $\left(15.5 \% \mathrm{P}_{2} \mathrm{O}_{5}\right)$ at 300 $\mathrm{kg} / \mathrm{fed} /$ year and potassium sulphate $(48 \%$ $\mathrm{K}_{2} \mathrm{O}$ ) at $100 \mathrm{~kg} / \mathrm{fed} /$ year, respectively.

All $\mathrm{P}$ amounts were added during preparing the soil to cultivation, while the mineral NK fertilizers were divided into four batches, two batches (the first and second

Table a. Physical and chemical analysis of the experimental soil.

\begin{tabular}{|c|c|c|c|}
\hline Soil Characters & Values & Soil Characters & Values \\
\hline Sand \% & 28.20 & Available P \% & 15.12 \\
\hline Silt \% & 30.70 & Exch. $\mathrm{K}^{+}(\mathrm{mg} / \mathbf{1 0 0} \mathrm{g})$ & 2.11 \\
\hline Clay \% & 41.10 & Exch. $\mathrm{Ca}^{++}(\mathrm{mg} / 100 \mathrm{~g})$ & 31.74 \\
\hline Soil type & Clayey loam & Exch. $\mathrm{Na}^{+}(\mathrm{mg} / \mathbf{1 0 0} \mathrm{g})$ & 2.40 \\
\hline Organic matter \% & 1.62 & $\mathrm{Fe}$ & 8.54 \\
\hline $\mathrm{CaCO}_{3} \%$ & 2.09 & $\mathrm{Cu}$ & 2.06 \\
\hline E.C. $(\mathbf{m m h o s} / \mathrm{cm})$ & 1.04 & DTPA & 2.75 \\
\hline pH (1:2.5) & 7.82 & Ext.ppm & 8.26 \\
\hline Total N\% & 0.08 & & \\
\hline
\end{tabular}


doses) were added at three weeks interval, starting from March $21^{\text {st }}$ (for first cutting), and the other two batches (the third and fourth doses) were added at three weeks interval, starting from June $2^{\text {nd }}$ (after 7 days of the first cutting time).

Minia Azotein (Azotobacter strain) as a biofertilizer was added $\left(50 \mathrm{~cm}^{3} /\right.$ plant $)$ four times around the plants after one week from each addition of mineral $\mathrm{N}$ and $\mathrm{K}$. All other agricultural practices were carried out as usual in the region in the two experimental seasons.

2- Sub plots were control (spraying with a distilled water) and ascorbic acid at 50, 100 and $150 \mathrm{ppm}$ as a foliar spray four times for each season at the same schedule of mineral NK.

During each experimental season the plants were cut twice, the first cut was done on $25^{\text {th }}$ May, while, the second cut was done on $25^{\text {th }}$ September in the two growth seasons.

\section{Data recorded:}

Plant height $(\mathrm{cm})$, number of main branches/plant, herb fresh weight/plant ( $\mathrm{g} / \mathrm{cut}$ and $\mathrm{kg} / \mathrm{season}$ ) and herb fresh weight/fed (ton/fed).

\section{Essential oil determination:}

Determination of essential oil $\%$ in random samples obtained from the fresh herb of each treatment (after 48 hours from each cut) was carried out in each cut during the two seasons according to the method described by British Pharmacopoeia (1963).

The essential oil percentage $=$ Essential oil in the graduated tube in $\mathrm{ml} /$ Weight of sample $(\mathrm{g}) \times 100$.

Essential oil yield/plant was calculated by multiplying oil percentage by herb yield/plant and expressed as $\mathrm{ml} /$ plant.

Essential oil yield/fed was calculated by essential oil yield/plant $\times$ number of plants/fed (14222.22 plants).

The data of the two cuts during two seasons were subjected to statistical analysis of variance MSTAT-C (1986). L.S.D. test at 0.05 was found to compare the average means of treatments.

\section{RESULTS AND DISCUSSION}

\section{1- Vegetative growth parameters:}

Data presented in Tables (1, 2, 3 and 4) show that the vegetative growth traits (plant height, number of main branches/plant, herb fresh weight/plant/cut and /plant/season, as well as, herb fresh weight/fed) were increased by increasing mineral $\mathrm{N}$ in the presence of Minia Azotein till 75\% N. The fertilization with $100 \%$ mineral nitrogen gave the highest values in comparison with other treatments of bio-mineral nitrogen fertilization without significant differences detected between mineral $\mathrm{N}(100 \%)$ and Minia Azotein + mineral N $(75 \%)$ in the two cuts during both seasons. The response of plant growth in the present investigation as a result of application of mineral $\mathrm{N}$ fertilization was also found by Ram et al. (2003) and Ibrahim (2010) on geranium, Golcz et al. (2006) on sweet basil and Aziz and El-Ashry (2009) on lemon Balm. The beneficial effects of Minia Azotein (Nbiofertilizer treatments) on plant growth were obtained by Hemdan (2008) on anise, Helmy (2008) on black cumin and Abdou et al. (2014) on sweet basil.

Concerning the effect of ascorbic acid treatments, all previous studied characters were significantly increased in the two cuts during both seasons due to the use of the three concentrations of ascorbic acid in comparison with control. Among various treatments, the high concentrations (150 ppm) gave the highest values comparing with other two concentrations and control. Similar results were obtained by Ismail (2008) on black cumin and Abdou et al. (2009) on borage plant.

Results showed a significant interaction between Minia Azotein-mineral $\mathrm{N}$ and ascorbic acid treatments. The highest values over all were obtained due to fertilizing 
Table 1. Effect of nitrogen fertilization and ascorbic acid on plant height $(\mathrm{cm})$ of geranium (Pelargonium graveolens, L.) plants in the first and second cuts during the two seasons $(2012 / 2013$ and 2013/2014).

\begin{tabular}{|c|c|c|c|c|c|c|c|c|c|c|}
\hline \multirow{2}{*}{$\begin{array}{l}\text { N-fertilization } \\
\text { Ascorbic acid } \\
\text { treatments }\end{array}$} & \multicolumn{5}{|c|}{$1^{\text {st }}$ Season } & \multicolumn{5}{|c|}{$2^{\text {nd }}$ Season } \\
\hline & $\begin{array}{c}\text { M.A. } \\
+ \\
25 \% \\
\text { N }\end{array}$ & $\begin{array}{c}\text { M.A. } \\
+ \\
\mathbf{5 0 \%} \mathrm{N}\end{array}$ & $\begin{array}{c}\text { M.A. } \\
+ \\
75 \% \mathrm{~N}\end{array}$ & $100 \% \mathrm{~N}$ & $\begin{array}{c}\text { Mean } \\
\text { (B) }\end{array}$ & $\begin{array}{c}\text { M.A. } \\
+ \\
25 \% \mathrm{~N}\end{array}$ & $\begin{array}{c}\text { M.A. } \\
+ \\
\mathbf{5 0 \%} \mathbf{N}\end{array}$ & $\begin{array}{c}\text { M.A. } \\
+ \\
75 \% \mathrm{~N}\end{array}$ & $100 \% \mathrm{~N}$ & $\begin{array}{c}\text { Mean } \\
\text { (B) }\end{array}$ \\
\hline \multicolumn{11}{|c|}{$1^{\text {st }}$ Cut } \\
\hline Control & 51.4 & 55.6 & 59.8 & 60.1 & 56.7 & 57.1 & 62.5 & 65.5 & 66.6 & 62.9 \\
\hline AsA. at 50 ppm & 55.1 & 59.3 & 62.2 & 63.2 & 60.0 & 61.2 & 67.0 & 69.3 & 70.2 & 66.9 \\
\hline AsA. at 100 ppm & 59.0 & 63.3 & 66.1 & 67.0 & 63.9 & 65.3 & 71.4 & 73.5 & 74.0 & 71.1 \\
\hline AsA. at $150 \mathrm{ppm}$ & 60.8 & 65.4 & 68.1 & 69.0 & 65.8 & 67.5 & 73.5 & 75.5 & 76.0 & 73.1 \\
\hline Mean (A) & 54.1 & 60.9 & 64.1 & 64.8 & & 62.8 & 68.6 & 71.0 & 71.7 & \\
\hline L.S.D. at 5\% & \multicolumn{2}{|c|}{$\mathrm{A}=2.8$} & $B=0.9$ & \multicolumn{2}{|c|}{$\mathrm{AB}=1.8$} & \multicolumn{2}{|c|}{$\mathrm{A}=2.2$} & $\mathrm{~B}=0.8$ & \multicolumn{2}{|c|}{$\mathrm{AB}=1.6$} \\
\hline \multicolumn{11}{|c|}{$2^{\text {nd }}$ Cut } \\
\hline Control & 48.1 & 51.2 & 53.3 & 54.1 & 51.7 & 53.1 & 58.1 & 60.8 & 61.5 & 58.4 \\
\hline AsA. at $50 \mathrm{ppm}$ & 51.2 & 54.7 & 56.5 & 57.2 & 54.9 & 57.0 & 62.0 & 64.5 & 65.5 & 62.3 \\
\hline AsA. at 100 ppm & 55.3 & 58.5 & 60.5 & 61.1 & 58.9 & 60.8 & 66.0 & 68.1 & 69.3 & 66.1 \\
\hline AsA. at $150 \mathrm{ppm}$ & 57.0 & 60.6 & 62.4 & 62.7 & 60.7 & 62.8 & 68.1 & 70.2 & 71.3 & 68.1 \\
\hline Mean (A) & 52.9 & 56.3 & 58.2 & 58.8 & & 58.4 & 63.6 & 65.9 & 66.9 & \\
\hline L.S.D. at $5 \%$ & \multicolumn{2}{|c|}{$\mathrm{A}=1.7$} & $\mathrm{~B}=0.7$ & \multicolumn{2}{|c|}{$\mathrm{AB}=1.4$} & \multicolumn{2}{|c|}{$\mathrm{A}=2.1$} & $\mathrm{~B}=0.9$ & \multicolumn{2}{|c|}{$\mathrm{AB}=1.8$} \\
\hline
\end{tabular}

AsA.= Ascorbic acid M.A.= Minia Azotein (Azotobacter strain)

Table 2. Effect of nitrogen fertilization and ascorbic acid on number of main branches/plant of geranium (Pelargonium graveolens, L.) plants in the first and second cuts during the two seasons $(2012 / 2013$ and $2013 / 2014)$.

\begin{tabular}{|c|c|c|c|c|c|c|c|c|c|c|}
\hline \multirow{2}{*}{$\begin{array}{l}\mathrm{N} \text {-fertilization } \\
\text { Ascorbic acid } \\
\text { treatments }\end{array}$} & \multicolumn{5}{|c|}{$1^{\text {st }}$ Season } & \multicolumn{5}{|c|}{$2^{\text {nd }}$ Season } \\
\hline & $\begin{array}{c}\text { M.A. } \\
+ \\
25 \% \\
\end{array}$ & $\begin{array}{c}\text { M.A. } \\
+ \\
\mathbf{5 0 \%} \mathrm{N}\end{array}$ & $\begin{array}{c}\text { M.A. } \\
+ \\
\mathbf{7 5} \% \mathrm{~N}\end{array}$ & $100 \% \mathrm{~N}$ & $\begin{array}{c}\text { Mean } \\
\text { (B) }\end{array}$ & $\begin{array}{c}\text { M.A. } \\
+ \\
25 \% \mathrm{~N}\end{array}$ & $\begin{array}{c}\text { M.A. } \\
+ \\
\mathbf{5 0 \%} \mathbf{N}\end{array}$ & $\begin{array}{c}\text { M.A. } \\
+ \\
\mathbf{7 5 \%}\end{array}$ & $100 \% \mathrm{~N}$ & $\begin{array}{c}\text { Mean } \\
\text { (B) }\end{array}$ \\
\hline \multicolumn{11}{|c|}{$1^{\text {st }} \mathrm{Cut}$} \\
\hline Control & 9.9 & 12.1 & 13.6 & 14.5 & 12.5 & 10.9 & 13.2 & 14.2 & 14.7 & 13.3 \\
\hline AsA. at 50 ppm & 11.1 & 13.4 & 14.7 & 15.2 & 13.6 & 12.3 & 14.7 & 15.8 & 16.1 & 14.7 \\
\hline AsA. at $100 \mathrm{ppm}$ & 11.4 & 13.7 & 15.0 & 15.2 & 13.8 & 12.6 & 14.9 & 16.0 & 16.0 & 14.9 \\
\hline AsA. at $150 \mathrm{ppm}$ & 11.7 & 13.7 & 15.4 & 15.6 & 14.1 & 12.7 & 15.0 & 16.1 & 16.5 & 15.0 \\
\hline Mean (A) & 11.0 & 13.2 & 14.7 & 15.1 & & 12.1 & 14.5 & 15.5 & 15.8 & \\
\hline L.S.D. at 5\% & \multicolumn{2}{|c|}{$\mathrm{A}=0.5$} & $\mathrm{~B}=0.1$ & \multicolumn{2}{|c|}{$\mathrm{AB}=0.2$} & \multicolumn{2}{|c|}{$\mathrm{A}=0.4$} & $\mathrm{~B}=0.2$ & \multicolumn{2}{|c|}{$\mathrm{AB}=0.4$} \\
\hline \multicolumn{11}{|c|}{$2^{\text {nd }} \mathrm{Cut}$} \\
\hline Control & 10.5 & 12.6 & 14.0 & 14.5 & 12.9 & 11.4 & 13.8 & 14.7 & 15.1 & 13.8 \\
\hline AsA. at 50 ppm & 11.9 & 13.8 & 15.0 & 15.4 & 14.0 & 12.7 & 15.1 & 16.0 & 16.4 & 15.1 \\
\hline AsA. at $100 \mathrm{ppm}$ & 12.4 & 14.4 & 15.7 & 16.1 & 14.7 & 13.3 & 15.8 & 16.8 & 17.0 & 15.7 \\
\hline AsA. at $150 \mathrm{ppm}$ & 12.8 & 14.9 & 16.3 & 16.7 & 15.2 & 13.4 & 15.9 & 17.2 & 17.4 & 16.0 \\
\hline Mean (A) & 11.9 & 13.9 & 15.3 & 15.7 & & 12.7 & 15.2 & 16.2 & 16.6 & \\
\hline L.S.D. at $5 \%$ & \multicolumn{2}{|c|}{$\mathrm{A}=0.5$} & $\mathrm{~B}=0.2$ & \multicolumn{2}{|c|}{$\mathrm{AB}=0.4$} & \multicolumn{2}{|c|}{$\mathrm{A}=0.6$} & $\mathrm{~B}=0.1$ & \multicolumn{2}{|c|}{$\mathrm{AB}=0.2$} \\
\hline
\end{tabular}


Table 3. Effect of nitrogen fertilization and ascorbic acid on herb fresh weight/plant (g/plant) of geranium (Pelargonium graveolens, L.) plants in the first and second cuts during the two seasons $(2012 / 2013$ and $2013 / 2014)$.

\begin{tabular}{|c|c|c|c|c|c|c|c|c|c|c|}
\hline \multirow{2}{*}{$\begin{array}{l}\text { N-fertilization } \\
\text { Ascorbic acid } \\
\text { treatments }\end{array}$} & \multicolumn{5}{|c|}{$1^{\text {st }}$ Season } & \multicolumn{5}{|c|}{$2^{\text {nd }}$ Season } \\
\hline & $\begin{array}{c}\text { M.A. } \\
+ \\
25 \% \mathrm{~N}\end{array}$ & $\begin{array}{c}\text { M.A. } \\
+ \\
\mathbf{5 0 \%} \mathbf{N}\end{array}$ & $\begin{array}{c}\text { M.A. } \\
+ \\
75 \% \mathrm{~N}\end{array}$ & $100 \% \mathrm{~N}$ & $\begin{array}{c}\text { Mean } \\
\text { (B) }\end{array}$ & $\begin{array}{c}\text { M.A. } \\
+ \\
25 \% \mathrm{~N}\end{array}$ & $\begin{array}{c}\text { M.A. } \\
+ \\
\mathbf{5 0 \%} \mathrm{N}\end{array}$ & $\begin{array}{c}\text { M.A. } \\
+ \\
75 \% \mathrm{~N}\end{array}$ & $100 \% \mathrm{~N}$ & $\begin{array}{c}\text { Mean } \\
\text { (B) }\end{array}$ \\
\hline \multicolumn{11}{|c|}{$1^{\text {st }} \mathrm{Cut}$} \\
\hline Control & 490.1 & 588.2 & 687.0 & 697.5 & 615.7 & 551.9 & 657.2 & 761.6 & 768.3 & 684.8 \\
\hline AsA. at 50 ppm & 593.1 & 710.2 & 825.8 & 841.8 & 742.7 & 605.1 & 719.3 & 838.2 & 850.9 & 753.4 \\
\hline AsA. at $100 \mathrm{ppm}$ & 620.0 & 741.1 & 868.5 & 900.5 & 782.5 & 629.1 & 753.2 & 879.5 & 910.0 & 793.0 \\
\hline AsA. at $150 \mathrm{ppm}$ & 641.1 & 773.5 & 910.6 & 935.8 & 815.3 & 648.2 & 780.1 & 918.8 & 943.5 & 822.7 \\
\hline Mean (A) & 586.1 & 703.3 & 823.0 & 843.9 & & 608.6 & 727.5 & 849.5 & 868.2 & \\
\hline L.S.D. at 5\% & \multicolumn{2}{|c|}{$\mathrm{A}=21.9$} & $\mathrm{~B}=12.9$ & \multicolumn{2}{|c|}{$\mathrm{AB}=25.8$} & \multicolumn{2}{|c|}{$\mathrm{A}=19.7$} & $\mathrm{~B}=12.4$ & \multicolumn{2}{|c|}{$\mathrm{AB}=24.8$} \\
\hline \multicolumn{11}{|c|}{$2^{\text {nd }}$ Cut } \\
\hline Control & 532.1 & 643.2 & 761.5 & 768.0 & 676.2 & 581.1 & 672.1 & 793.2 & 797.5 & 711.1 \\
\hline AsA. at 50 ppm & 617.1 & 741.2 & 865.6 & 871.3 & 773.8 & 626.2 & 750.1 & 874.2 & 880.1 & 782.7 \\
\hline AsA. at $100 \mathrm{ppm}$ & 651.1 & 781.0 & 930.2 & 944.5 & 826.7 & 658.9 & 790.3 & 937.5 & 952.6 & 834.8 \\
\hline AsA. at $150 \mathrm{ppm}$ & 672.0 & 817.0 & 955.3 & 971.3 & 853.9 & 683.2 & 826.1 & 964.8 & 980.1 & 863.6 \\
\hline Mean (A) & 618.1 & 745.6 & 878.2 & 888.8 & & 637.4 & 759.7 & 892.5 & 902.6 & \\
\hline L.S.D. at 5\% & \multicolumn{2}{|c|}{$\mathrm{A}=13.6$} & $B=9.0$ & \multicolumn{2}{|c|}{$\mathrm{AB}=18.0$} & \multicolumn{2}{|c|}{$\mathrm{A}=14.5$} & $\mathrm{~B}=8.1$ & \multicolumn{2}{|c|}{$\mathrm{AB}=16.2$} \\
\hline
\end{tabular}

AsA.= Ascorbic acid M.A.= Minia Azotein (Azotobacter strain)

Table 4. Effect of nitrogen fertilization and ascorbic acid on herb fresh weight/plant/season (kg/plant) and herb fresh weight/fed/season (ton/fed) of geranium (Pelargonium graveolens, L.) plants in the first and second cuts during the two seasons $(2012 / 2013$ and $2013 / 2014)$.

\begin{tabular}{|c|c|c|c|c|c|c|c|c|c|c|}
\hline \multirow{2}{*}{$\begin{array}{l}\text { N-fertilization } \\
\text { Ascorbic acid } \\
\text { treatments }\end{array}$} & \multicolumn{5}{|c|}{$1^{\text {st }}$ Season } & \multicolumn{5}{|c|}{$2^{\text {nd }}$ Season } \\
\hline & $\begin{array}{c}\text { M.A. } \\
+ \\
25 \% \\
\end{array}$ & $\begin{array}{c}\text { M.A. } \\
+ \\
\mathbf{5 0 \%} \mathbf{N}\end{array}$ & $\begin{array}{c}\text { M.A. } \\
+ \\
75 \% \\
75\end{array}$ & $100 \% \mathrm{~N}$ & $\begin{array}{c}\text { Mean } \\
\text { (B) }\end{array}$ & $\begin{array}{c}\text { M.A. } \\
+ \\
25 \% \\
\text { N }\end{array}$ & $\begin{array}{c}\text { M.A. } \\
+ \\
\mathbf{5 0 \%} \mathbf{N}\end{array}$ & $\begin{array}{c}\text { M.A. } \\
+ \\
\mathbf{7 5 \%}\end{array}$ & $100 \% \mathrm{~N}$ & $\begin{array}{c}\text { Mean } \\
\text { (B) }\end{array}$ \\
\hline \multicolumn{11}{|c|}{ Herb fresh weight/plant/season (kg/plant) } \\
\hline Control & 1.02 & 1.23 & 1.45 & 1.47 & 1.29 & 1.13 & 1.33 & 1.56 & 1.57 & 1.40 \\
\hline AsA. at 50 ppm & 1.21 & 1.45 & 1.69 & 1.71 & 1.52 & 1.23 & 1.47 & 1.71 & 1.73 & 1.54 \\
\hline AsA. at $100 \mathrm{ppm}$ & 1.27 & 1.52 & 1.80 & 1.85 & 1.61 & 1.29 & 1.54 & 1.82 & 1.86 & 1.63 \\
\hline AsA. at $150 \mathrm{ppm}$ & 1.31 & 1.59 & 1.87 & 1.91 & 1.67 & 1.33 & 1.61 & 1.88 & 1.92 & 1.69 \\
\hline Mean (A) & 1.20 & 1.45 & 1.70 & 1.74 & & 1.25 & 1.49 & 1.74 & 1.77 & \\
\hline L.S.D. at $5 \%$ & \multicolumn{2}{|c|}{$\mathrm{A}=0.05$} & $\mathrm{~B}=0.02$ & \multicolumn{2}{|c|}{$\mathrm{AB}=0.04$} & \multicolumn{2}{|c|}{$\mathrm{A}=0.04$} & $\mathrm{~B}=0.02$ & \multicolumn{2}{|c|}{$\mathrm{AB}=0.04$} \\
\hline \multicolumn{11}{|c|}{ Herb fresh weight/fed/season (ton/fed) } \\
\hline Control & 14.51 & 17.49 & 20.62 & 20.91 & 18.35 & 16.07 & 18.92 & 22.19 & 22.33 & 19.91 \\
\hline AsA. at 50 ppm & 17.21 & 20.62 & 24.04 & 24.32 & 21.62 & 17.49 & 20.91 & 24.32 & 24.60 & 21.90 \\
\hline AsA. at $100 \mathrm{ppm}$ & 18.06 & 21.62 & 25.60 & 26.31 & 22.90 & 18.35 & 21.90 & 25.88 & 26.45 & 23.18 \\
\hline AsA. at $150 \mathrm{ppm}$ & 18.63 & 22.61 & 26.60 & 27.16 & 23.75 & 18.92 & 22,90 & 26.74 & 27.31 & 24.04 \\
\hline Mean (A) & 17.07 & 20.62 & 24.18 & 24.75 & & 17.78 & 21.19 & 24.75 & 25.17 & \\
\hline L.S.D. at $5 \%$ & \multicolumn{2}{|c|}{$\mathrm{A}=0.59$} & $B=0.30$ & \multicolumn{2}{|c|}{$\mathrm{AB}=0.60$} & \multicolumn{2}{|c|}{$\mathrm{A}=0.46$} & $\mathrm{~B}=0.32$ & \multicolumn{2}{|c|}{$\mathrm{AB}=0.64$} \\
\hline
\end{tabular}


geranium plants with either mineral $\mathrm{N}$ $(100 \%)$ or Minia Azotein + mineral N (75\%) and spraying plants with ascorbic acid at 150 ppm.

\section{2- Essential oil productivity:}

The data presented in Tables (5, 6 and 7) indicate that essential oil $\%$, essential oil yield/plant/cut, essential oil yield/plant and /fed were significantly decreased as a result of fertilizing plants with all used combined fertilization treatments (mineral $\mathrm{N}+$ Minia Azotein) comparing with mineral N (100\%). It is noticed that the least reduction of values was obtained with mineral N (75\%) + Minia Azotein and there were non-significant differences between $100 \% \mathrm{~N}$ and $75 \% \mathrm{~N}+$ Minia Azotein for all previous parameters.

Data presented in Tables (5, 6 and 7) indicated that the treatments of ascorbic acid (50, 100 and $150 \mathrm{ppm})$ significantly increased essential oil percentage, essential oil yield (per plant/cut, per plant/season and per fed) over the control. Ascorbic acid at
$150 \mathrm{ppm}$ was more effective than other treatments. Ascorbic acid has been known as an antioxidant and protects plants against damage resulting from aerobic metabolism, photosynthesis and a range of pollutants. Also, it had promotive effects on essential oil \% (Tarraf et al., 1999).

The interaction between fertilization and ascorbic acid treatments was significant for essential oil \% and essential oil yield (per plant and per fed) in both seasons. The best interaction in this study was obtained from either fertilizing geranium plants with mineral nitrogen $(100 \%)$ or with Minia Azotein $+75 \% \mathrm{~N}$ and spraying plants with salicylic acid at $150 \mathrm{ppm}$.

Hence, it could be recommended that the interaction treatments of Minia Azotein + $75 \% \mathrm{~N}$ (mineral) plus ascorbic acid at 150 ppm had a beneficial effect on growth and essential oil production of geranium plants.

Table 5. Effect of nitrogen fertilization and ascorbic acid on essential oil \% of geranium (Pelargonium graveolens, L.) plants in the first and second cuts during the two seasons (2012/2013 and 2013/2014).

\begin{tabular}{|c|c|c|c|c|c|c|c|c|c|c|}
\hline \multirow{2}{*}{$\begin{array}{l}\text { N-fertilization } \\
\text { Ascorbic acid } \\
\text { treatments }\end{array}$} & \multicolumn{5}{|c|}{$1^{\text {st }}$ Season } & \multicolumn{5}{|c|}{$2^{\text {nd }}$ Season } \\
\hline & $\begin{array}{c}\text { M.A. } \\
+ \\
25 \% \mathrm{~N}\end{array}$ & $\begin{array}{c}\text { M.A. } \\
+ \\
\mathbf{5 0 \%} \mathbf{N}\end{array}$ & $\begin{array}{c}\text { М.A. } \\
+ \\
\mathbf{7 5 \%} \mathbf{N}\end{array}$ & $100 \% \mathrm{~N}$ & $\begin{array}{c}\text { Mean } \\
\text { (B) }\end{array}$ & $\begin{array}{c}\text { M.A. } \\
+ \\
25 \% \mathrm{~N}\end{array}$ & $\begin{array}{c}\text { M.A. } \\
+ \\
\mathbf{5 0 \%} \mathbf{N}\end{array}$ & $\begin{array}{c}\text { M.A. } \\
+ \\
\mathbf{7 5 \%} \mathbf{N}\end{array}$ & $100 \% \mathrm{~N}$ & $\begin{array}{c}\text { Mean } \\
\text { (B) }\end{array}$ \\
\hline \multicolumn{11}{|c|}{$1^{\text {st }} \mathrm{Cut}$} \\
\hline Control & 0.331 & 0.346 & 0.374 & 0.378 & 0.357 & 0.333 & 0.348 & 0.377 & 0.380 & 0.360 \\
\hline AsA. at 50 ppm & 0.351 & 0.356 & 0.387 & 0.388 & 0.371 & 0.353 & 0.368 & 0.399 & 0.401 & 0.380 \\
\hline AsA. at 100 ppm & 0.357 & 0.363 & 0.394 & 0.398 & 0.378 & 0.362 & 0.378 & 0.409 & 0.413 & 0.391 \\
\hline AsA. at 150 ppm & 0.365 & 0.372 & 0.401 & 0.404 & 0.386 & 0.369 & 0.385 & 0.416 & 0.419 & 0.397 \\
\hline Mean (A) & 0.351 & 0.359 & 0.389 & 0.392 & & 0.354 & 0.370 & 0.400 & 0.403 & \\
\hline L.S.D. at 5\% & \multicolumn{2}{|c|}{$\mathrm{A}=0.004$} & $\mathrm{~B}=0.002$ & \multicolumn{2}{|c|}{$\mathrm{AB}=0.004$} & \multicolumn{2}{|c|}{$\mathrm{A}=0.004$} & $\mathrm{~B}=0.002$ & \multicolumn{2}{|c|}{$\mathrm{AB}=0.004$} \\
\hline \multicolumn{11}{|c|}{$2^{\text {nd }} \mathrm{Cut}$} \\
\hline Control & 0.319 & 0.333 & 0.363 & 0.366 & 0.545 & 0.325 & 0.337 & 0.369 & 0.372 & 0.351 \\
\hline AsA. at 50 ppm & 0.338 & 0.353 & 0.384 & 0.387 & 0.366 & 0.348 & 0.360 & 0.395 & 0.397 & 0.375 \\
\hline AsA. at 100 ppm & 0.345 & 0.360 & 0.391 & 0.394 & 0.373 & 0.354 & 0.367 & 0.401 & 0.404 & 0.382 \\
\hline AsA. at 150 ppm & 0.351 & 0.366 & 0.398 & 0.399 & 0.379 & 0.364 & 0.377 & 0.413 & 0.416 & 0.393 \\
\hline Mean (A) & 0.338 & 0.353 & 0.384 & 0.387 & & 0.348 & 0.360 & 0.395 & 0.397 & \\
\hline L.S.D. at 5\% & \multicolumn{2}{|c|}{$\mathrm{A}=0.003$} & $\mathrm{~B}=0.002$ & \multicolumn{2}{|c|}{$\mathrm{AB}=0.004$} & \multicolumn{2}{|c|}{$\mathrm{A}=0.002$} & $\mathrm{~B}=0.005$ & \multicolumn{2}{|c|}{$\mathrm{AB}=0.010$} \\
\hline
\end{tabular}


Table 6. Effect of nitrogen fertilization and ascorbic acid on essential oil yield/plant/cut (ml/plant) of geranium (Pelargonium graveolens, L.) plants in the first and second cuts during the two seasons (2012/2013 and 2013/2014).

\begin{tabular}{|c|c|c|c|c|c|c|c|c|c|c|}
\hline \multirow{2}{*}{$\begin{array}{l}\mathrm{N} \text {-fertilization } \\
\text { Ascorbic acid } \\
\text { treatments }\end{array}$} & \multicolumn{5}{|c|}{$1^{\text {st }}$ Season } & \multicolumn{5}{|c|}{$2^{\text {nd }}$ Season } \\
\hline & $\begin{array}{c}\text { M.A. } \\
+ \\
\mathbf{2 5 \%} \mathbf{N} \\
\end{array}$ & $\begin{array}{c}\text { M.A. } \\
+ \\
\mathbf{5 0 \%} \mathrm{N}\end{array}$ & $\begin{array}{c}\text { M.A. } \\
+ \\
75 \% \mathrm{~N}\end{array}$ & $100 \% \mathrm{~N}$ & $\begin{array}{c}\text { Mean } \\
\text { (B) }\end{array}$ & $\begin{array}{c}\text { M.A. } \\
+ \\
25 \% \mathrm{~N}\end{array}$ & $\begin{array}{c}\text { M.A. } \\
+ \\
\mathbf{5 0 \%} \mathrm{N}\end{array}$ & $\begin{array}{c}\text { M.A. } \\
+ \\
\mathbf{7 5 \%} \mathbf{N}\end{array}$ & $100 \% \mathrm{~N}$ & $\begin{array}{c}\text { Mean } \\
\text { (B) }\end{array}$ \\
\hline \multicolumn{11}{|c|}{$1^{\text {st }} \mathrm{Cut}$} \\
\hline Control & 1.62 & 2.04 & 2.57 & 2.64 & 2.20 & 1.84 & 2.29 & 2.87 & 2.92 & 2.47 \\
\hline AsA. at 50 ppm & 2.08 & 2.53 & 3.20 & 3.27 & 2.76 & 2.14 & 2.65 & 3.34 & 3.41 & 2.86 \\
\hline AsA. at 100 ppm & 2.21 & 2.69 & 3.42 & 3.58 & 2.96 & 2.28 & 2.85 & 3.60 & 3.76 & 3.10 \\
\hline AsA. at 150 ppm & 2.34 & 2.88 & 3.65 & 3.78 & 3.15 & 2.39 & 3.00 & 3.82 & 3.95 & 3.27 \\
\hline Mean (A) & 2.06 & 2.52 & 3.20 & 3.31 & & 2.15 & 2.69 & 3.40 & 3.50 & \\
\hline L.S.D. at 5\% & \multicolumn{2}{|c|}{$\mathrm{A}=0.13$} & $B=0.07$ & \multicolumn{2}{|c|}{$\mathrm{AB}=0.14$} & \multicolumn{2}{|c|}{$\mathrm{A}=0.12$} & $\mathrm{~B}=0.07$ & \multicolumn{2}{|c|}{$\mathrm{AB}=0.14$} \\
\hline \multicolumn{11}{|c|}{$2^{\text {nd }} \mathrm{Cut}$} \\
\hline Control & 1.70 & 2.14 & 2.76 & 2.81 & 3.69 & 1.89 & 2.26 & 2.93 & 2.97 & 2.50 \\
\hline AsA. at 50 ppm & 2.09 & 2.62 & 3.32 & 3.37 & 2.83 & 2.18 & 2.70 & 3.45 & 3.49 & 2.94 \\
\hline AsA. at 100 ppm & 2.25 & 2.81 & 3.64 & 3.72 & 3.08 & 2.33 & 2.90 & 3.76 & 3.85 & 3.19 \\
\hline AsA. at 150 ppm & 2.36 & 2.99 & 3.80 & 3.88 & 3.24 & 2.49 & 3.11 & 3.98 & 4.08 & 3.39 \\
\hline Mean (A) & 2.09 & 2.63 & 3.37 & 3.44 & & 2.22 & 2.73 & 3.53 & 3.58 & \\
\hline L.S.D. at 5\% & \multicolumn{2}{|c|}{$\mathrm{A}=0.11$} & $B=0.06$ & \multicolumn{2}{|c|}{$\mathrm{AB}=0.12$} & \multicolumn{2}{|c|}{$\mathrm{A}=0.11$} & $\mathrm{~B}=0.11$ & \multicolumn{2}{|c|}{$\mathrm{AB}=0.22$} \\
\hline
\end{tabular}

AsA.= Ascorbic acid $\quad$ M.A.= Minia Azotein (Azotobacter strain)

Table 7. Effect of nitrogen fertilization and ascorbic acid on essential oil yield/plant (ml/plant) and essential oil yield/fed (liter/fed) of geranium (Pelargonium graveolens, L.) plants in the first and second cuts during the two seasons (2012/2013 and 2013/2014).

\begin{tabular}{|c|c|c|c|c|c|c|c|c|c|c|}
\hline \multirow{2}{*}{$\begin{array}{l}\text { N-fertilization } \\
\text { Ascorbic acid } \\
\text { treatments }\end{array}$} & \multicolumn{5}{|c|}{$1^{\text {st }}$ Season } & \multicolumn{5}{|c|}{$2^{\text {nd }}$ Season } \\
\hline & $\begin{array}{c}\text { M.A. } \\
+ \\
25 \% \mathrm{~N}\end{array}$ & $\begin{array}{c}\text { M.A. } \\
+ \\
\mathbf{5 0 \%} \mathbf{N}\end{array}$ & $\begin{array}{c}\text { М.A. } \\
+ \\
\mathbf{7 5 \%} \mathbf{N}\end{array}$ & $100 \% \mathrm{~N}$ & $\begin{array}{c}\text { Mean } \\
\text { (B) }\end{array}$ & $\begin{array}{c}\text { M.A. } \\
+ \\
25 \% \mathrm{~N}\end{array}$ & $\begin{array}{c}\text { M.A. } \\
+ \\
\mathbf{5 0 \%} \mathbf{N}\end{array}$ & $\begin{array}{c}\text { M.A. } \\
+ \\
75 \% \mathrm{~N}\end{array}$ & $100 \% \mathrm{~N}$ & $\begin{array}{c}\text { Mean } \\
\text { (B) }\end{array}$ \\
\hline \multicolumn{11}{|c|}{ Essential oil yield/plant (ml/plant) } \\
\hline Control & 3.32 & 4.18 & 5.33 & 5.45 & 5.88 & 3.73 & 4.55 & 5.80 & 5.89 & 4.96 \\
\hline AsA. at 50 ppm & 4.17 & 5.14 & 6.52 & 6.64 & 5.59 & 4.32 & 5.35 & 6.80 & 6.91 & 5.80 \\
\hline AsA. at 100 ppm & 4.46 & 5.50 & 7.06 & 7.31 & 6.04 & 4.61 & 5.75 & 7.36 & 7.61 & 6.29 \\
\hline AsA. at 150 ppm & 4.70 & 5.87 & 7.45 & 7.66 & 6.38 & 4.88 & 6.12 & 7.81 & 8.03 & 6.66 \\
\hline Mean (A) & 4.15 & 5.16 & 6.57 & 6.75 & & 4.37 & 5.43 & 6.92 & 7.08 & \\
\hline L.S.D. at 5\% & \multicolumn{2}{|c|}{$\mathrm{A}=0.20$} & $\mathrm{~B}=0.11$ & \multicolumn{2}{|c|}{$\mathrm{AB}=0.22$} & \multicolumn{2}{|c|}{$\mathrm{A}=0.18$} & $\mathrm{~B}=0.12$ & \multicolumn{2}{|c|}{$\mathrm{AB}=0.24$} \\
\hline \multicolumn{11}{|c|}{ Essential oil yield/fed (liter/fed) } \\
\hline Control & 47.2 & 59.4 & 75.9 & 77.5 & 83.7 & 53.0 & 64.7 & 82.5 & 83.7 & 70.6 \\
\hline AsA. at 50 ppm & 59.3 & 73.2 & 92.7 & 94.4 & 79.5 & 61.4 & 76.1 & 96.7 & 98.2 & 82.5 \\
\hline AsA. at 100 ppm & 63.4 & 78.2 & 100.4 & 103.9 & 85.9 & 65.6 & 81.7 & 104.6 & 108.2 & 89.5 \\
\hline AsA. at 150 ppm & 66.8 & 83.5 & 106.0 & 108.9 & 90.8 & 69.4 & 87.0 & 111.0 & 114.2 & 94.7 \\
\hline Mean (A) & 59.0 & 73.3 & 93.5 & 96.0 & & 62.2 & 77.2 & 98.5 & 100.7 & \\
\hline L.S.D. at 5\% & \multicolumn{2}{|c|}{$\mathrm{A}=2.6$} & $B=2.4$ & \multicolumn{2}{|c|}{$\mathrm{AB}=4.8$} & \multicolumn{2}{|c|}{$\mathrm{A}=2.4$} & $\mathrm{~B}=2.8$ & \multicolumn{2}{|c|}{$\mathrm{AB}=5.6$} \\
\hline
\end{tabular}

AsA.= Ascorbic acid $\quad$ M.A.= Minia Azotein (Azotobacter strain) 


\section{REFERENCES}

Abdou, M.A.H.; Aly, M.K.; Zaki, K.A.; Sadek, A.A. and El-Husseiny, R. (2009). Response of borage plants to some organic and biofertilization treatments. Proc. $5^{\text {th }}$ of Sustain, Agric. and Develop. Fac. of Agric., Fayoum Univ. 21-23 December, pp:143-158.

Abdou, M.A.H.; Badran, F.S.; El-Sayed, A.A.; Taha, R.A. and Abd-El-Salam, N.M.K. (2014). Response of sweet basil plants to some agricultural treatments. Minia J. Agric. Res. \& Develop., 34(1):21-31.

Aziz, E.E. and El-Ashry, S.M. (2009). Efficiency of slow release urea fertilizer on herb yield and essential oil production of lemon balm (Melissa officinalis, L.) plant. American-Eurasian J. Agric. \& Environ. Sci., 5(2):141-147.

British Pharmacopoeia(1963). Determination of Volatile Oil Drugs. The Pharmaceutical Press, London.

Dewick, P.M. (2000). Medicinal Natural Products: A Biosynthetic Approach $2^{\text {nd }}$ Ed., John Wiley \& Sons, N, Y., pp:306356.

Galal, Y.G.M. and Aly, B.E. (2004). IIBiofertilization and Organic Farming Approaches. Advances in Agric. Res. in Egypt. Special Issue Vol. S, N.I.P. 99176. Published by the Agric. Res. Center, Giza, Egypt.

Golcz, A.; Politycka, B. and Lozykowska, S. (2006). The effect of nitrogen fertilization and stage of plant development on the mass and quality of sweet basil leaves (Ocimum basilicum, L.). ERBA Polonica, 52(112):22-30.

Helmy, T.A. (2008). Effect of Some Organic and Biofertilization Treatments on Black Cumin Plants. M.Sc. Thesis, Fac. Agric., Minia Univ., Egypt.

Hemdan, S.H.O. (2008). Effect of Some Organic and Biofertilization Treatments on Anise Plants. M.Sc. Thesis, Fac. Agric. Minia Univ. Egypt.

Ibrahim, T.I.E. (2010). Physiological Studies on Geranium Plants. M.Sc. Thesis, Fac. Agric. Minia Univ., Egypt.

Ismail, S.I.I. (2008). Anatomical and Physiological Studies on Nigella sativa, L. Plant. Ph.D. Thesis., Fac. Agric., Mansoura Univ., Egypt.

Kaul, P.N.; Rajeshwara, B.R.; Bhattachary, A.K.; Mallavarapu, G. R. and Ramesh, S. (1996). Changes in chemical composition of rose-scented geranium (Pelargonium $\mathrm{sp)}$ oil during storage. J. Ess. Oil Res., 9:115.

MSTAT-C (1986). A Microcomputer Program for the Design, Management and Analysis of Agronomic Research Experiments (Version 4.0), Michigan Stat Univ., U.S.

Qinghua, Z. (1993). China's perfumery industry picks up. Perfum. Flavor., 18:297-299.

Rajeshwara Rao, B.R. (2002). Biomass yield, essential oil yield and essential oil composition of rose scented geranium (Pelargonium sp.) as influenced by row spacing and intercropping with corn mint. Ind. Crops. Prod., 16:133-144.

Ram, M.; Ram, D. and Roy, S.K. (2003). Influence of an organic mulching on fertilizer nitrogen use efficiency and herb and essential oil yields in geranium (Pelargonium graveolens). Bioresource Technology, 87:273-278.

Shawi, A.S.; Kumar, T.; Chist, N. and Shabir, S. (2006). Cultivation of rose scented geranium (Pelargonium sp.) as a cash crop in kashmir valley. Asian J. of Plant Sciences, 5(41):673-675.

Tarraf, Sh.A.; Gamal El-Din, K.M. and Balbaa, L.K. (1999). The response of vegetative growth, essential oil of lemongrass (Cymbopogon citratus Hort.) to foliar application of ascorbic acid, 
nicotinamide and some micronutrients. Arab Universities J. of Agric. Sci. 7(1):247-259. (C.F. Hort. Abst. 1999, 69(11):9827).

Zheljazkov, V.D. and Cerven, V. (2009).

Effect of nitrogen, location and harvesting stage on peppermint productivity, oil content and oil composition. Hort. Sci., 44(5):12671270 .

تأثير مصادر النتروجين و حمض الأسكوربيك على النمو وإنتاج الزيت الطيار في نباتات العتر

محمود عبد الهادي حسن عبد" و عماد الدين توفيق أحمد" و طارق إبراهيم الاسوقي إسماعيل إبراهيم "*

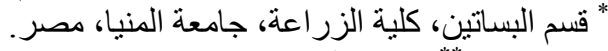

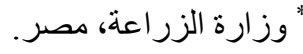

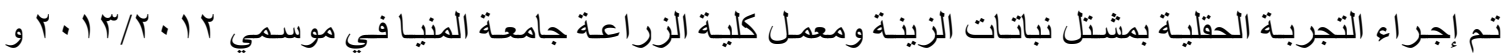

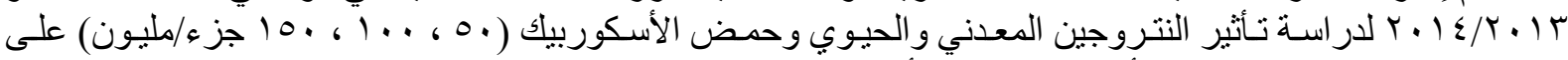

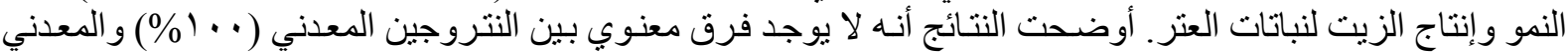

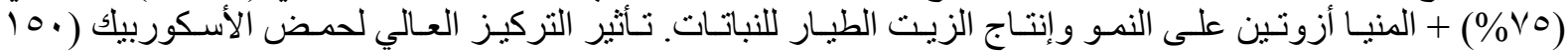

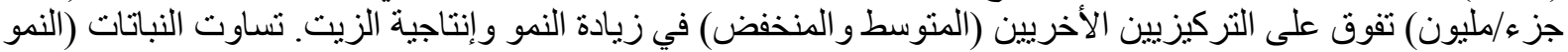

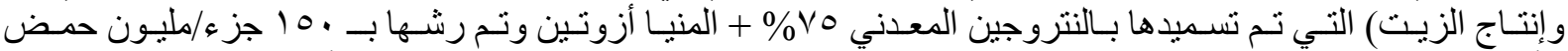

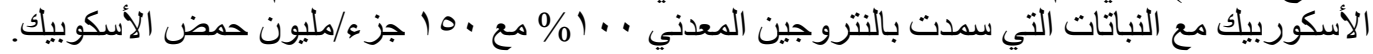

